\title{
Factors affecting bacterial propagation towards tumor microenvironments
}

\begin{abstract}
This paper provides a detailed analysis to identify factors that improve bacterial targeting times to tumor microenvironments. We utilize the "active flagellar" motion characteristic of the bacterial movement to present a continuous time Markov model and a first passage time analysis to determine the factors that affect the mean time taken for injected bacteria to reach a tumor micro environment. We have determined 3 major factors that play a role in determining mean targeting times: (i) the population of the injected bacteria, (ii) the distance between the region of injection and the tumor microenvironment and (iii) the directional efficiency of the injected bacteria. Of these 3 factors our results show that the directional efficiency is the most significant factor that affects the time taken by different bacterial species to reach the tumor micro environment. While distance between the tumor micro environment and the region of injection also plays an important role, population of bacteria is most ineffective, especially for large directional efficiency. Our model matches well with some existing experimental results.
\end{abstract}

Keywords: targeting time, tumor microenvironment engineered bacteria, markov model, first passage time
Volume 4 Issue I - 2016

\author{
Anand S,' Sujatha P Koduvayur, ${ }^{2}$ R \\ Chandramouli \\ 'Department of Electrical Engineering, New York Institute of \\ Technology, USA \\ ${ }^{2}$ Department of Electrical and Computer Engineering, Stevens \\ Institute of Technology, USA
}

\begin{abstract}
Correspondence: Sujatha P Koduvayur, Department of Electrical and Computer Engineering, Stevens Institute of Technology, NJ 07030, USA, Email skoduvay@stevens.edu
\end{abstract}

Received: September 09, 2016 | Published: September 29, 2016
Abbreviations: DDS, drug delivery systems; EPR, enhanced permeability and retention; CTMC, continuous time markov chain

\section{Introduction}

Drug delivery systems (DDS) directly affect the effectiveness of a drug's pharmacokinetics, absorption, metabolism, duration of effect and toxicity. ${ }^{1}$ DDS can be broadly classified into passive and active targeting. Passive targeting is solely dependent on Enhanced Permeability and Retention (EPR) effect ${ }^{2,3}$ Active targeting on the other hand can be of two types; (i) homing to disease sites by loading targeting agents with ligands or antibodies that bind specifically to biomarkers uniquely expressed/secreted in diseased regions ${ }^{4}$ or (ii) self-propelling drug carriers. ${ }^{5}$ EPR is a direct effect of poor and leaky vasculature that is normally seen in disease sites (e.g., tumor microenvironments) and is only effective for upto $10 \%$ retention of therapeutics at these sites. ${ }^{6}$ While antibody and ligand directed nanoparticles show high levels of specific uptake by diseased cells, their targeting rates were still found to be low, as their accumulation in target sites $(<10 \%)$ is still dependent on the EPR effect. ${ }^{7,8}$ Recently, molecular communication based models were developed to optimize antibody based drug delivery systems. ${ }^{9,10}$ The current state of the art investigates various remote-controlled delivery strategies to overcome poor localization of drug carriers to disease sites. Ultrasonic waves and light directed targeting of nanoparticles to disease sites have been recently investigated and show great promise for remote-controlled DDS. ${ }^{11-15}$ Despite these promising advances, self-propelling/autonomous swimmers remain the most encouraging active DDS platforms.

Bacteria, the classic example of self-propelling/autonomous swimmers, move towards or away from external/environmental signals by a process called cheomotaxis. ${ }^{16,17}$ Anaerobic bacteria (bacteria that grow in low/no oxygen sites e.g. tumors) have been used as anti-cancer agents since Coley's experiments in 1890s. ${ }^{18}$
Since then great strides have been taken to improve and adapt bacterial DDS to a great many diseases. Currently more than 50 patents have been issued to use different strains of bacteria as anti-cancer agents. ${ }^{19}$ Bacteria are idea active targeting DDS as they can actively penetrate tissues, home in to disease sites in response to disease-specific biomarkers extruded into the extracellular space, and controllably induce cytotoxicity. ${ }^{20}$ More significantly, bacteria can grow selectively in disease tissue thereby compensating for poor targeting..$^{21}$ Bacteria have been used to deliver anti-cancer drugs or nanoparticles and to deliver genes intracellular. ${ }^{22,23}$ Successful tumor regression studies in mice ${ }^{24}$ and other laboratory animals have led to recent FDA approved clinical trials of bacterial targeted anticancer treatments. ${ }^{25-27}$ These trials showed that patients tolerated upto $10^{8} \mathrm{c}$.f.u $/ \mathrm{m}^{2}$ of bacteria in the system without any adverse effects by either intravenous injection or intra-tumoral injection respectively. Nemunaitis ${ }^{27}$ further showed that these bacteria were able to specifically target an enzyme that could catalyze the conversion of a well known anti-cancer prodrug to its active, killing state. However, these trials showed very poor colonization of the tumors in situ with the best-case scenario being one colony per tumor. Thus, recent research has focused on improving bacterial targeting strategies to tumor sites. Thornlow et al., ${ }^{28}$ show that increasing the motility of anerobic bacteria, Salmonella and generating homogeneous population of highly motile Salmonella, enhanced tumor penetration in vitro.

The chemo taxis capability and their effectiveness in DDS motivated the study of diffusion models for bacteria. A continuous diffusion model was developed ${ }^{29}$ to characterize the diffusion of bacterial colonies. Recent research has focused on improving bacterial targeting strategies to tumor sites. Thornlow et al. ${ }^{28}$ show that increasing the motility of anerobic bacteria, Salmonella and generating homogeneous population of highly motile Salmonella, enhanced tumor penetration in vitro. Okaie et al., ${ }^{30}$ developed a microsensor network equivalent of bacterial 
chemotaxis. They exploited the model in ${ }^{31}$ (later extended in) $)^{32}$ where bacteria were modeled as microsensors and base stations were placed to monitor the different sensors.

The fact that tumor targeting bacteria not just diffuse but demonstrate sensing based motility towards the tumor ${ }^{33}$ (called "active flagellar" motion) gives rise to the need to develop stochastic models for bacterial propagation towards tumors. Some key researches on stochastic modeling of bacteria for chemotherapy are very recent. ${ }^{34-36}$ Charteris \& Khain $^{34}$ developed a discrete stochastic lattice model to model the cells in the body as a combination of discrete lattices and the velocity of bacteria between successive lattices. The model was verified with experiments and it was shown that bacteria typically propagate only unidirectional to neighboring lattices. Lavrentovich \& Nelson $^{35}$ and Deng ${ }^{36}$ separately studied the time for survival of bacteria in any geographic region using diffusion model ${ }^{35}$ and by colonial communication mode ${ }^{36,37}$ and showed that the time spent in any geographical region is exponentially distributed. Therefore, the propagation of engineered bacteria can be modeled as a stochastic random walk. ${ }^{36,37}$ However, the nature of these findings has not yet been exploited to analyze the time taken for bacteria to reach tumor microenvironments and determine the factors that impact this time. Wei et al., ${ }^{37}$ presented simulations on first passage time to tumors. A diffusion equation was formulated that used spatial extensions to Gillespei's algorithm. ${ }^{38}$

Motivated by the findings in, ${ }^{34-36}$ we divide the region of propagation of the bacteria ${ }^{1}$ into a 3-dimensional lattice and develop a three-dimensional Markov Chain model to characterize the random walk behavior of the bacteria between the grids in the lattice. We use first passage time analysis of Markov processes ${ }^{2}$ to provide closed form expressions for the mean time taken for the bacteria to reach a desired proximity to the tumor, from the region (i.e., grid) of injection. From the first passage time analysis of Markov processes, we derive the following key results:

- The main factors that affect the tumor targeting time (time taken for the bacteria to reach the tumor microenvironment from the point of injection) are (a) the population of the injected bacteria, (b) the distance between the tumor and the region of injection and (c) the directional efficiency, defined as the ratio of the rate at which bacteria move towards the tumor microenvironment to the rate at which they move away from the tumor microenvironment.

The directional efficiency is the most significant factor that impacts time taken to reach the tumor microenvironment. While the distance between the tumor microenvironment and the region of injection also play a significant role, the population is the least effective parameter, particularly beyond a certain threshold value (called the saturation threshold of population). The results obtained from out model matches well with existing experimental results ${ }^{28}$ that measure the time taken by control bacteria to reach the tumor microenvironment. To the best of our knowledge, this is the first research that characterizes the first passage times of injected bacteria reaching the tumor microenvironment, to clearly bring out the dependencies on the population, directional efficiency of the bacteria and the distance between the region of injection and the tumor. Our analysis can be extended to these scenarios at the cost of additional

${ }^{1}$ While the concept of Markov models and first pass time was introduced and discussed in, ${ }^{36}$ the first pass times discussed were very crude approximations that took only the speed of the bacteria into account.

${ }^{2}$ Henceforth, throughout the paper, whenever we refer to "'bacteria", we mean "injected engineered bacteria", unless explicitly mentioned otherwise. computational complexity.

The rest of the paper is organized as follows. Section 5 defines the objective of this research and builds the Markov model. Numerical results are described in Section 6 and conclusions are drawn in Section 7.

\section{Problem description}

Consider a finite population of engineered bacteria injected into a medium (e.g., blood stream), bound for a tumor. Initially, at the point of injection, the bacteria are unaware of the exact location of the tumor thus they propagate according to a random walk model. ${ }^{34-37}$ Once the bacteria reach the tumor microenvironment, they then move towards the tumor, by active chemotaxis in response to chemical signal from the tumor ${ }^{17,29}$ and grow according to the model discussed in. ${ }^{33}$ The objective is to estimate the mean time taken by the bacteria to reach the tumor microenvironment from the point of injection. For this, we develop a Markov model that characterizes the random walk behavior from the point of injection to the tumor microenvironment. We first describe the basic system model and the assumptions we make, in Section 5.1 and the Markov model in Section 5.2.

\section{a. System model}

We divide the medium into set of rectangular lattices in threedimension based on the lattice model in. ${ }^{34}$. For simplicity, a two dimensional equivalent is shown in Figure 1. The lattice division shown in Figure 1 is only notional and does not depict a real-life representation of the medium. The grids shown in Figure 1 only represent a region where a bacterium might be present at any instant of time ${ }^{3}$. We make the following model description to facilitate the problem definition and the related analysis.

- The bacteria is injected in a region labeled by the gird $(0,0$, $0)$ in three dimensions (the gird, $(0,0)$ in the two-dimensional representation shown in Figure 1). Irrespective of where the bacteria are physically injected into the body, we consider the region of injection to be represented by the gird, $(0,0,0)$.

- The negative indices for the grids represent regions that are opposite in direction from the region of injection with respect to the tumor microenvironment. In general, the negative indices can go up to -of, but for purposes of computation and tractability, we limit them to $-\mathrm{m}$. It is noted that since the negative indices represent propagation away from the desired target region, the values of negative indices are not relevant for our analysis, as will be observed later from (8).

- The grid, (m, m, m) (or the grid, (m, m) in the two dimensional representation shown in Figure 1) depicts the tumor microenvironment. This does not represent the actual tumor. It only represents the outer bounds of the region surrounding the tumor beyond which chemical signals from the tumor do not reach. Within the bounds of the tumor microenvironment (past grid $(\mathrm{m}, \mathrm{m}, \mathrm{m})$ or $(\mathrm{m}, \mathrm{m})$ in Figure 1) the bacteria propagate to the tumor by chemotaxis..$^{17,29}$

- Note that although we have shown the regions to be of uniform size, they are not so in practice. However, the variations in the sizes of the individual regions can be easily incorporated by suitably changing the number of regions, $\mathrm{m}$.

${ }^{3}$ Later, from Theorem 2.1, it will be observed that the dimensions of the gird and the number if such grids do not affect the time taken to reach the tumour microenvironment. 
- Until the bacteria reach the grid $(\mathrm{m}, \mathrm{m}, \mathrm{m})$ (or $(\mathrm{m}, \mathrm{m})$ in the twodimensional representation shown in Figure 1), they follow a random walk behavior. ${ }^{34-36}$

- The objective is to develop a stochastic model that can be applied to determine the mean time taken to reach the tumor microenvironment from the region of injection, i.e., the time taken to reach grid $(\mathrm{m}, \mathrm{m}, \mathrm{m})$ starting from grid, $(0,0,0)$. This model should provide: the parameters that affect the mean time taken to reach $(\mathrm{m}, \mathrm{m}, \mathrm{m})$ starting from $(0,0,0)$ and - the factors that are more dominant, i.e., that have more significant effect on the mean time, compared to other factors.

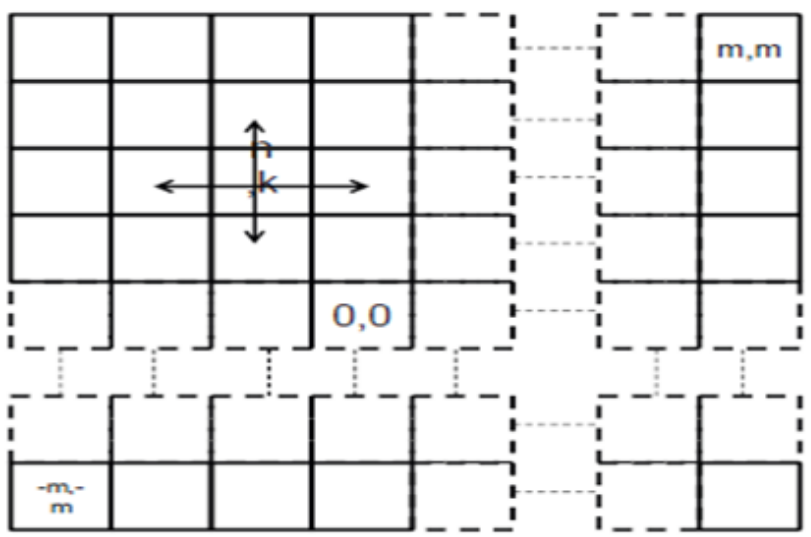

Figure I A grid representation of the bacterial propagation region. For simplicity a two-dimensional picture is shown. In our model we use 3-dimensional lattice.

Bacteria can move only to one of four neighbouring cells (either in the vertical or horizontal direction but not diagonally. ${ }^{34}$ The region, $(0 ; 0)$, represents the point at which the bacteria is injected and $(\mathrm{m} ; \mathrm{m})$ represents the tumor microenvironment.

\section{b. Markov model}

Until, the bacteria injected in region depicted by the grid $(0$, $0,0)$ reach the tumor microenvironment, i.e., until the bacteria reach the region, $(\mathrm{m}, \mathrm{m}, \mathrm{m})$, they move according to a random walk process ${ }^{36,37}$ by spending a random time in each of the regions shown in Figure 1. The random time is exponentially distributed. ${ }^{35}$ Therefore, we model the random walk behavior of the bacteria from the region of injection $(0,0,0)$ until it reaches $(\mathrm{m}, \mathrm{m}, \mathrm{m})$ as a three-dimensional continuous time Markov chain (3D-CTMC). In general, a bacteria in grid $(\mathrm{n}, \mathrm{k}, \mathrm{l})(-m, \leq \mathrm{n}, \mathrm{k}, \mathrm{l} \leq \mathrm{m})$, can move to a grid $(\mathrm{n}, \mathrm{k}, \mathrm{l})$, where

$$
\begin{aligned}
& \max (-m, n-1) \leq \tilde{n} \leq \min (\mathrm{m}, \mathrm{n}+1) \\
& \max (-m, \mathrm{k}-1) \leq \tilde{k} \leq \min (\mathrm{m}, \mathrm{k}+1) \\
& \max (-m, 1-1) \leq \tilde{l} \leq \min (\mathrm{m}, 1+1)
\end{aligned}
$$

Based on the finding in,${ }^{34}$ the velocity or motility in the diagonal directions (i.e., when $i^{\prime}=i \pm 1$ and $j^{\prime}=j \pm 1$ was zero. Hence, bacteria can only move to the regions to the left, right, up or down. In a three dimensional lattice, the six possible directions a bacteria can move to would be left, right, front, back, up and down. For simplicity, in Figure 2, we show the state diagram for a $2 \mathrm{D}-\mathrm{CTMC}$ assuming that the region is split into an $\mathrm{m} \times \mathrm{m}$ grid in two-dimensions as shown in Figure 1. Let $(n ; k ; 1)$ represent any state of a CTMC, i.e., represents the region at which a bacteria is present. The transition rate, $\mathrm{q}_{n k \tilde{k} \tilde{n} \tilde{k}]}$ represents that rate at which the CTMC transits from state, $(\mathrm{n}, \mathrm{k}, 1)$ to state, $\pi \tilde{n} \tilde{k} \tilde{l}$ Then

$$
\mathrm{q}_{n k l \tilde{n} \tilde{k} \tilde{l}}=\left\{\begin{array}{l}
\lambda \tilde{n}=n+1, \tilde{k}=k, \tilde{l}=1-m \leq n, k, l<m \\
\lambda \tilde{n}=n, \tilde{k}=k+1, \tilde{l}=l-m \leq n, k, l<m \\
\lambda \tilde{n}=n, \tilde{k}=k, \tilde{l}=l+1-m \leq n, k, l<m \\
\mu \tilde{n}=n-1, \tilde{k}=k, \tilde{l}=l-m<n, k, l \leq m \\
\mu \tilde{n}=n, \tilde{k}=k-1, \tilde{l}=l-m<n, k, l \leq m \\
\mu \tilde{n}=n, k=k, \tilde{l}=l-1-m<n, k, l \leq m \\
\mathrm{O}
\end{array}\right.
$$

\section{Otherwise}

This constitutes a three dimensional birth-death process. Since the movement of the bacteria follows a random walk, the direction of transition from any state to its neighboring state is independent of the direction of the previous transition. Therefore, the steady state probability of being in state $(\mathrm{n}, \mathrm{k}, 1), 7 \mathrm{rnki}$, is obtained by applying the analysis in $^{39}$ as

$$
\pi_{n k l}=G \prod_{\tilde{n}=-m}^{n-1} \prod_{\tilde{k}=-m \tilde{l}=-m}^{k-1} \prod_{\tilde{n}}^{l-1} \Delta_{\tilde{k}} \Delta_{\tilde{l}}
$$

Where $\Delta_{\tilde{n}}=\frac{\lambda_{\tilde{n}}}{\mu_{\tilde{n}+1}} ; \lambda_{\tilde{n}}$ is the rate of transiting from state $\tilde{n} \tilde{k} \tilde{l}$ to $(\tilde{n}+1, \tilde{k} \tilde{l})$ and $\mu_{\tilde{n}+1}$ is the rate of transiting from $(\tilde{n}+1, \tilde{k} \tilde{l})$ to $\tilde{n}, \tilde{k}, \tilde{l}$ ( $\Delta_{\bar{k}}$ and $\Delta_{\bar{k}}$ are also defined similarly). The factor, G, in (2) is a normalization factor, so that $\sum_{n, k, l} \pi_{n k l=1}$. For 3-D equivalent of the transition diagram shown in Figure 2, $\Delta_{\tilde{n}=\Delta_{\tilde{k}}=\Delta_{\tilde{l}}=\rho=\frac{\lambda}{\mu}}$ i.e.,

$$
\pi_{n k l}=\rho^{n+k+l}\left(\frac{1-\rho}{1-\rho^{2 m+1}}\right)^{3}-m \leq n, k, l \leq m
$$

The ratio, $\rho \frac{\lambda}{\mu}$ is the ratio of the rate at which the bacteria move towards the tumor to that at which they move away from the tumor, i.e., the directional efficiency of the bacteria. The average time taken to reach the region $(\mathrm{m}, \mathrm{m}, \mathrm{m})$ starting from state, $(0,0,0)$ is obtained by first-pass time analysis of a birthdeath process. Let $\tilde{U}(\mathrm{~m})$ represent the average time taken for the CTMC to reach $(\mathrm{m}, \mathrm{m}, \mathrm{m})$ starting from state, $(0,0,0)$. Let $\tilde{U}$ $(\mathrm{m}, 0,0)^{4}$ represents the average first pass time to reach state $(\mathrm{m}$, $0,0)$ from state $(0,0,0)$ in a one dimensional birth-death process. Formally, $\tilde{U}(\mathrm{~m}, 0,0)$ can be written as

$$
\tilde{u}(m, 0,0))=E\left[\inf _{s \geq 0} \tilde{X}(s)=(m, 0,0) \mid \tilde{\mathrm{X}}(0)=(0,0,0,)\right]
$$

where $\bar{X}(\mathrm{t})$ is the grid in which the bacteria are present at time, t. The Laplace transform of $\tilde{U}(1 ; 0 ; 0)$

is denoted by $\tilde{f}_{1}(s)$, given by ${ }^{40}$

$$
\tilde{f}_{1}(s)=\frac{\mu}{s+\lambda+\mu}+\frac{\lambda}{s+\lambda+\mu} \tilde{f}_{2}(s) \tilde{f}_{1}(s),
$$

${ }^{4} \tilde{U}(m, 0,0)$ and $\tilde{U}(0,0, m)$ can be similarly defined. 
which can be solved recursively. Then,

$$
\tilde{u}(m, 0,0)=\lim _{s \rightarrow 0}-\frac{d \tilde{f}_{1}(s)}{d s} .
$$

Since the transition rates from any state, $(\mathrm{n}, \mathrm{k}, 1)$ to $(\mathrm{n}+1, \mathrm{k}, 1)$, (n, $\mathrm{k}+1,1)$ and $(\mathrm{n}, \mathrm{k}, 1+1)$ are all equal to $\lambda^{5}$

$$
\begin{aligned}
& \tilde{U}(0, m, 0)=\tilde{U}(0,0, m)=\tilde{U}(m, 0,0) \\
& \tilde{U}(m)=\tilde{U}(m, 0,0)+\tilde{U}(0, m, 0)+\tilde{U}(0,0, m) \\
& =3 \tilde{U}(m, 0,0) \\
& =\frac{3}{\lambda} \sum_{j=1}^{m} \frac{1}{\rho^{j-1}}\left(1+\sum_{i=1}^{j-1} \frac{\rho^{i}}{i}\right) .
\end{aligned}
$$

from (5)-(7). It is observed that the first pass time depends on $\mathrm{m}$. This implies that the time taken for the bacteria to reach the tumor microenvironment is a function of the number of lattice grids the region of propagation is divided into. Note that the number of lattice regions, $\mathrm{m}$, also impact the transition rates, $\lambda$ and $\mu$ since they are dependent on the average motility of the bacteria and the size of individual regions. The following theorem helps us remove the dependence on $\mathrm{m}$.
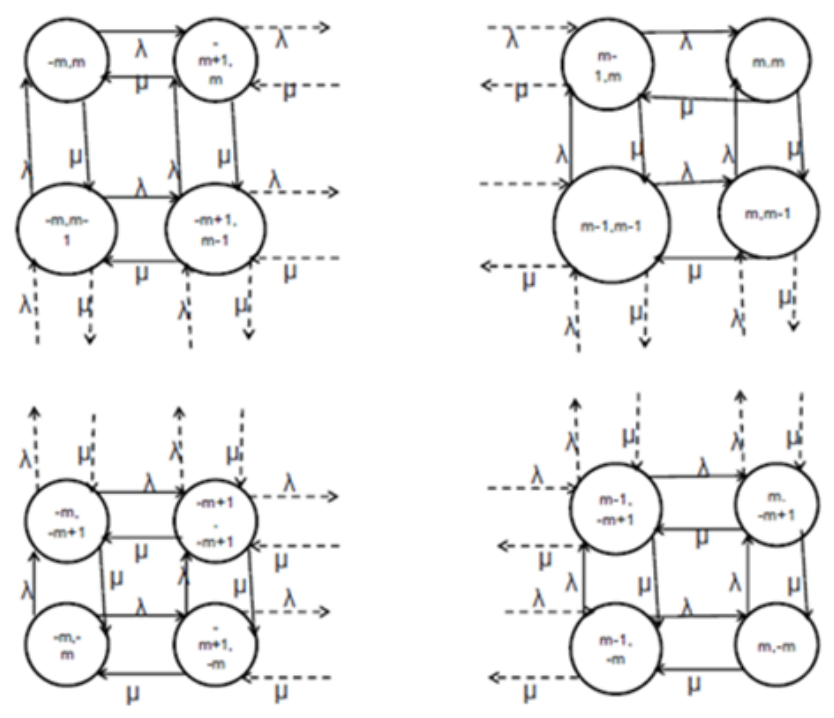

Figure 2 Two dimensional equivalent of the 3D CTMC model for random walk behavior of bacterial propagation.

The negative signs indicate grids away from the direction of the tumor, relative to the region of injection.

Theorem 2.1: When the directional efficiency and the number of grids are large, the first passage time to the tumor does not depend on the number of grids.

Proof: From (8),

$$
\lim _{m \rightarrow \infty} \tilde{U}(m)=\frac{3}{\lambda} \lim _{m \rightarrow \infty} \frac{3}{\lambda} \sum_{j=1}^{m} \frac{1}{\rho^{j-1}}\left(1+\sum_{i=1}^{j-1} \frac{\rho^{i}}{i}\right)
$$

5Similarly, transition from state, $(\mathrm{n}, \mathrm{k}, \mathrm{l})$ to states, $(\mathrm{n}-1, \mathrm{k}, 1),(\mathrm{n}, \mathrm{k}-1,1)$ and (n, k, 1- 1) are all equal to $\mu$ Using the fact that the movement of the bacteria follows a random walk, i.e., the direction of transition from any state to its neighboring state is independent of the direction of the previous transition, which simplifies to

$$
\lim _{m \rightarrow \infty} \tilde{U}(m)=\frac{3}{\lambda} \sum_{j=1}^{\infty} \frac{1}{\rho^{j-1}}+\frac{3}{\lambda} \sum_{j=1}^{\infty} \sum_{i=1}^{j-1}=\frac{\rho^{i}}{i \rho^{j-1}}=\frac{3}{\lambda} \frac{1}{1-\frac{1}{\rho}}+\frac{3}{\lambda} \sum_{i=1}^{\infty} \frac{1}{i \rho^{1}} \sum_{j=i+1}^{\infty} \frac{1}{\rho^{j-1}}
$$

When $\mathrm{p}>1$ (i.e., when the directional efficiency of the bacteria is large), the above simplifies to

$$
\lim _{m \rightarrow \infty} \tilde{U}(m)=\frac{3}{\lambda} \frac{p}{\rho-1}\left(1+\operatorname{In} \frac{\rho}{\rho-1}\right)
$$

which is independent of $\mathrm{m}$. Note that from Figure 2, the number of grids in three dimensions is $(2 m+1)^{3}$

Since, $\lim _{m \rightarrow \infty} \tilde{U}(m)$ is independent of $m$ from (9), it is independent of the number of grids, $(2 m+1)^{3}$

If the bacteria travels at velocity or motility, $v$, the distance between the region of injection of the bacteria and tumor microenvironment is $\mathrm{d}$ and the cell diameter is $\mathrm{c}$, then $d \sqrt{2} \times c \times m$, i.e., $m \approx\left\lceil\frac{d}{\sqrt{2 c}}\right\rceil$ and $\lambda \approx \frac{v}{d \sqrt{2 m}} \approx \frac{c v}{d^{2}}$, which is independent of $\mathrm{m}$. Also, if the approximation is ignored, $\lambda$ and $\mu$ vary with $m$ at the same rate and hence the ratio $\rho=\frac{\lambda}{\mu}$ becomes independent of $\mathrm{m}$. Therefore, the approximate time to reach the desired region is independent of the size of the lattice, $m$. However, if bacteria move in groups of population of size, $g$, then $\lambda=g \lambda_{g}$ where $\lambda_{g}$ is the rate of each individual in the group and $\tilde{U}(m)$ is inversely proportional to $\mathrm{g}$, indicating that as the population size increases, the time taken to reach the tumor microenvironment decreases.

\section{Results and discussion}

For the numerical computations, we consider cell diameter of $1.1 \mathrm{pm}^{34}$ and bacterial motility of $2.5 \mu \mathrm{m} / \mathrm{s}$ to $37.5 \mu \mathrm{m} / \mathrm{s},{ }^{28}$ i.e., about 2 to 34 cell diameters per second. This results in a $\lambda_{g}=\frac{2}{d^{2}}$ to $\lambda_{g}=\frac{300}{d^{2}}$ where $\mathrm{d}$ is the distance between the point of injection and the tumor microenvironment, expressed in microns. For a population of size, $g, \lambda=g \lambda_{g}$ as mentioned in Section 5.2. We compute the average time taken to reach the tumor microenvironment, as a function of the motility of the bacteria. We consider three cases,

The directional efficiency, $\rho \triangleq \frac{\lambda}{\mu}=\frac{1}{2}$ i.e., bacteria is twice as fast to move away from the tumor microenvironment as towards the tumor microenvironment.

- $\mathrm{p}=1$, i.e., the bacteria is equally likely to move away from the tumor microenvironment as well as towards the tumor microenvironment.

- $\mathrm{p}=2$, i.e., the bacteria is twice as fast to move towards the tumor microenvironment as away from the tumor microenvironment.

For each case above, we measure the average time taken to reach the tumor microenvironment from the region of injection, when the distance, $d$, from the region of injection to the 
tumor microenvironment (i) $\mathrm{d}=100 \mu \mathrm{m}$, (i) $\mathrm{d}=1000 \mu \mathrm{m}$ and (iii) $\mathrm{d}=10000 \mu \mathrm{m}$. Figures $3-5$ depict the first passage time results for $\rho=\frac{1}{2}$ and 2 , respectively.

It is observed from Figures 3-5, that there is a threshold population size (called saturation threshold) beyond which, the increase in population size is ineffective. From Figure 3, it is observed that when $\rho=\frac{1}{2}$, for an order of increase in $\mathrm{d}$, there is an order of increase in the time taken to reach the tumor microenvironment from the region of injection, e.g., from 15 hours for $\mathrm{d}=100 \mu \mathrm{m}$ (see Figure $3 \mathrm{a}$ ) to 150 hours for $\mathrm{d}=1000 \mu \mathrm{m}$ (Figure $3 \mathrm{~b}$ ) and to 1250 hours for $\mathrm{d}=10000 \mu \mathrm{m}$ (Figure $3 \mathrm{c}$ ) for a motility of $25 \mu \mathrm{m}$ $\left.\mathrm{s}^{-1}\right)$.

The saturation threshold is larger for smaller distances $(=10000$ for $\mathrm{d}=100 \mathrm{pm})$ while it reduces as $\mathrm{d}$ increases $(=4000$ for $\mathrm{d}=1000 \mu \mathrm{m}$ and $=2500$ for $\mathrm{d}=10000 \mu \mathrm{m})$. This indicates that if distance between the region of injection and the tumor increases, and then increase in population does not impact the first passage time to reach the tumor microenvironment. This is because, larger population is effective for smaller d because even when a subset of the population reaches the tumor microenvironment, it can draw the rest of the population towards the tumor microenvironment. However, when the distance, $d$, is large, the entire population is still far away from the tumor microenvironment, thereby negating the effect of population.

When the directional efficiency becomes larger i.e., when $\mathrm{p}=1$ (see Figure 4), the saturation threshold decreases, e.g., $=2000$ for $\mathrm{d}=100 \mu \mathrm{m}$ (from Figure $4 \mathrm{a}$ ) and $=1500$ for $\mathrm{d}=1000 \mu \mathrm{m}$ (Figure $4 \mathrm{~b}$ and $\mathrm{d}=10000 \mu \mathrm{m}$ (Figure $4 \mathrm{c}$ ). This is because, when the rate of going towards the tumor and away from the tumor are same, even a smaller population of bacteria find their way to the tumor and a larger population is not necessarily required. When $\mathrm{p}=1$, it is observed that an order of increase in d results in an increase in the first passage time by a factor of 4 to 5 (increase from 8 hours for $\mathrm{d}=100 \mu \mathrm{m}$ to 30 hours for $\mathrm{d}=1000 \mu \mathrm{m}$ and to 150 hours for $\mathrm{d}=10000 \mu \mathrm{m}$ for a motility of $25 \mathrm{pms}^{-1}$ ). Since the directional efficiency, $p$, increases, the bacteria find their way to the tumor better than when $\mathrm{p}=$ even when the distance between the region of injection and the tumor increases. The numerical values we obtain match well with existing experimental results in ${ }^{28}$ that show that control bacteria reach the tumor growing edge in about 8hours, in vitro, where the distance between the injection site of bacteria and tumor edge is about $150 \mu \mathrm{m}$.

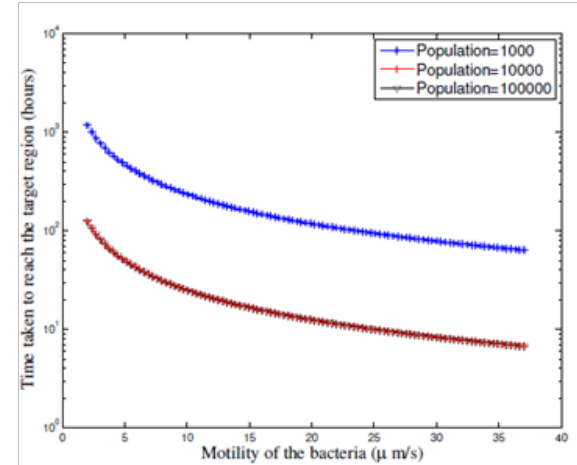

(a) $d=100 \mu \mathrm{m}$

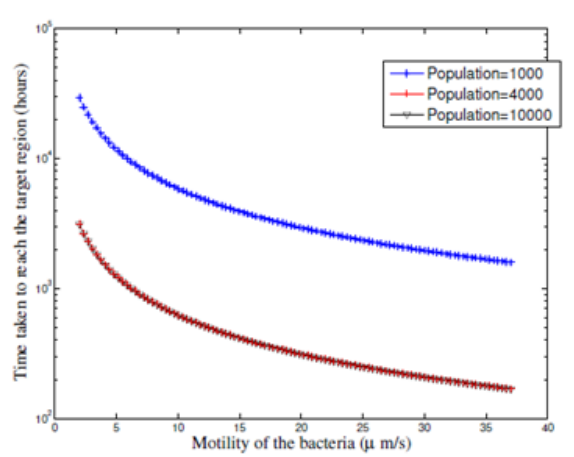

(b) $d=1000 \mu \mathrm{m}$

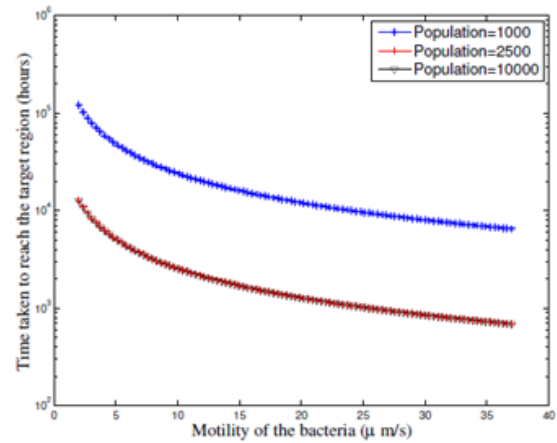

(c) $d=10000 \mu \mathrm{m}$

Figure 3 Average time taken to reach the tumor microenvironment from the region of injection $\left(U^{\sim}(\mathrm{m})\right.$ in $\left.(8)\right)$. Here the directional efficiency, $\rho \triangleq \lambda \mu=I 2$ beyond a certain threshold, (called saturation threshold), the population size is ineffective.

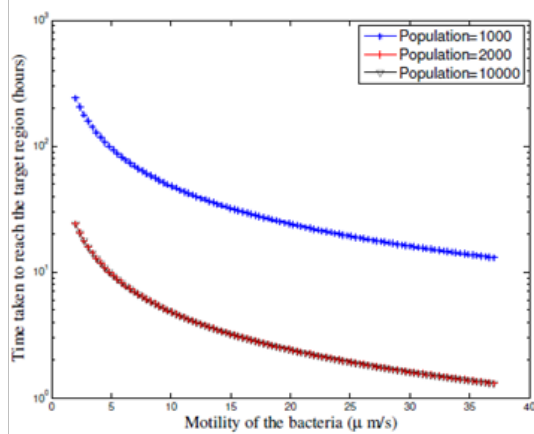

(a) $d=100 \mu \mathrm{m}$

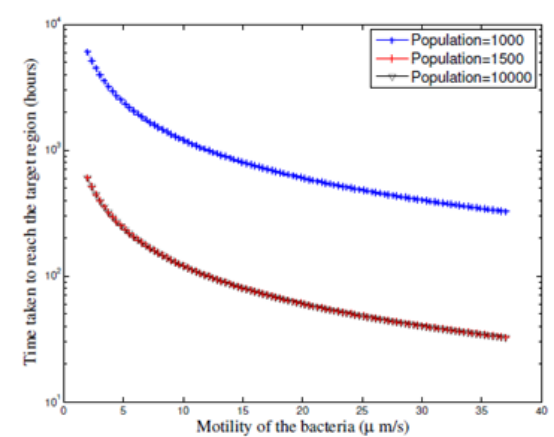

(b) $d=1000 \mu \mathrm{m}$

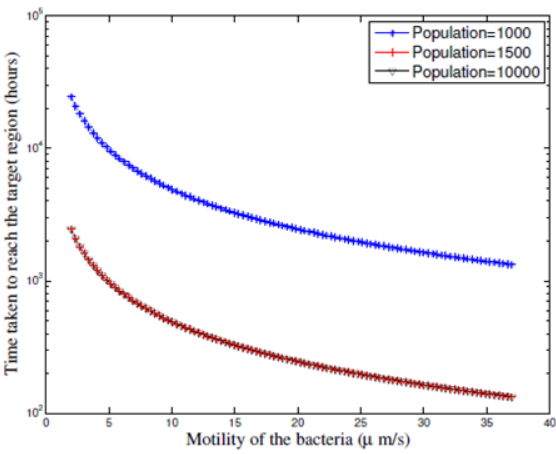

(c) $d=10000 \mu \mathrm{m}$

Figure 4 Average time taken to reach the tumor microenvironment from the region o injection $U^{r}(m)$ in $(8)$. Here the directional efficiency, $p \triangleq \lambda \mu=I$ compared to $p \triangleq \lambda \mu=\mid$ the average time taken to reach the tumour microenvironment is one order of magnitude less. The population saturation threshold is smaller. An order of increase in $d$ results in less than one order of increase in the first passage time ( a factor of 5$)$.

The saturation threshold decreases further then the directional efficiency, $\mathrm{p}=2$, as seen in Figure 5. The values are 1350 for $\mathrm{d}=100 \mu \mathrm{m}$ from Figure $5 \mathrm{a})$ and 1250 for $\mathrm{d}=1000 \mu \mathrm{m}$ and $\mathrm{d}=10000 \mu \mathrm{m}$ from Figure $5 \mathrm{~b}$ and $5 \mathrm{c}$. When $\mathrm{p}=2$, an order of increase in the distance from the region of injection to the tumor microenvironment results in an increase in the first passage 
time by a factor of 2 to 4 (increases from 2 hours for $\mathrm{d}=100 \mu \mathrm{m}$ to 8 hours for $\mathrm{d}=1000 \mu \mathrm{m}$ and 16 hours for $\mathrm{d}=10000 \mu \mathrm{m}$ for a motility of $25 \mathrm{pms}^{-1}$. A directional efficiency of $\mathrm{p}=2>1$ implies that even for large distances, small population of bacteria are twice as fast towards the tumor microenvironment than away from the tumor microenvironment, thereby leading to a reduction in the factor of increase in the first passage time and a reduction in saturation threshold.

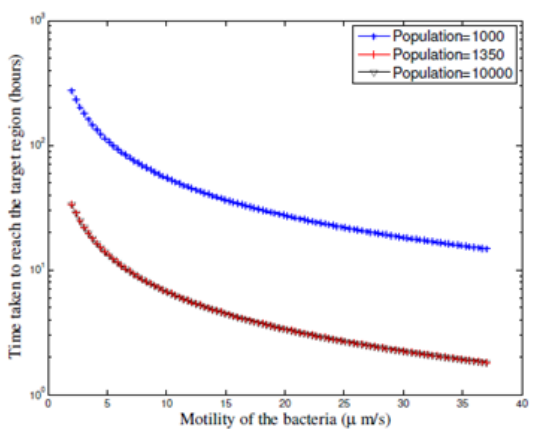

(a) $d=100 \mu \mathrm{m}$

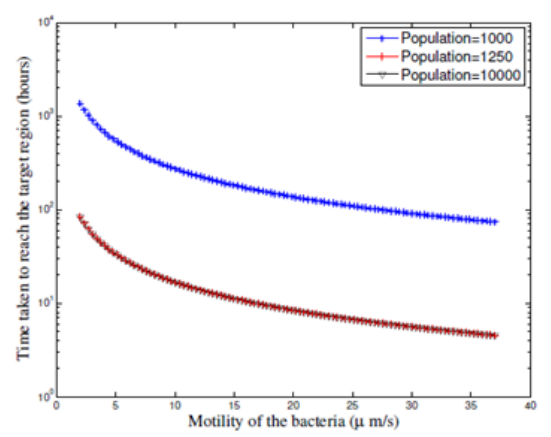

(b) $d=1000 \mu \mathrm{m}$

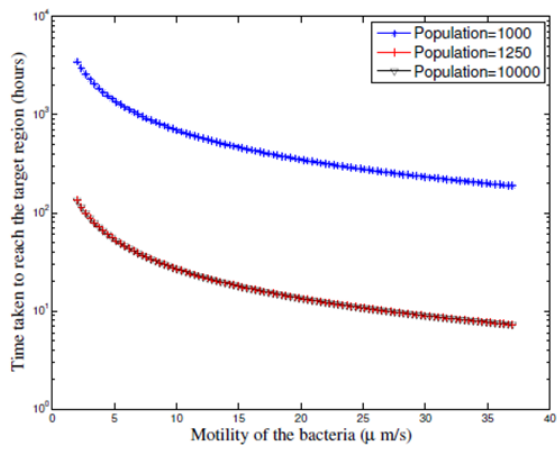

(c) $d=10000 \mu \mathrm{m}$

Figure 5 Average time taken to reach the tumour microenvironment from the region of injection $U(m)$ in (8). Here the directional efficiency, $p \triangleq \lambda \mu=2$. The saturation threshold is smaller than the case with $p=I$ an order of increase in $d$ results in an increase by a factor of 2 in the first passage time. This indicates that the effect of $p$ is more significant than the effect of distance and population size.

\section{Conclusion}

We presented a continuous time Markov model and a first passage time analysis to determine the time taken for bacteria to propagate towards tumor microenvironment. Key findings include:

- There is a threshold population (called saturation threshold) beyond which, the time taken by the bacteria to reach the tumor microenvironment is independent of bacterial population.

- Saturation threshold is larger when directional efficiency, $\mathrm{p}<1$ and smaller when $\mathrm{p}>1$.

- For smaller directional efficiency, an increase in an order of magnitude in the distance between the region of injection and the tumor results in an increase by an order of magnitude in the time taken to reach the tumor microenvironment.

- For larger directional efficiency, an order of increase in the distance between the region of injection and the tumor microenvironment results in an increase only by a factor of 2 to 5 in the time taken to reach the tumor microenvironment.

- Directional efficiency of the bacteria plays the most significant role in its impact on the time taken to reach the tumor microenvironment. While the distance between the region of injection and the tumor microenvironment also has significant impact on the time taken to reach the tumor, the population of injected bacteria plays the least significant roles.

Our analysis can be extended to heterogeneous bacteria by changing the transition rates in (1) to be unequal for different bacteria. Similarly, anisotropy can be taken into account by changing the rates in (1) for different directions. We are currently investigating the analysis in instances where distinct bacterial populations use quorum sensing signaling to enhance exchange of environmental information (e.g., as discussed in). ${ }^{30}$

\section{Acknowledgements}

None.

\section{Conflict of interest}

The author declares no conflict of interest.

\section{References}

1. Brunton LL, Gilman. The Pharmacological Basis of Therapeutics. 12th ed. USA: McGraw-Hill Medical; 2011. 2021 p.

2. Schroeder A, Sigal A, Turjeman K, et al. Using PEGylated nanoliposomes to target tissue invaded by a foreign body. J Drug Targeting. 2008;16(7):591-595.

3. Maeda H, Matsumura Y. EPR effect based drug design and clinical outlook for enhanced cancer chemotherapy. Adv Drug Delivery Rev. 2011;63(3):129-130.

4. Hrkach J, Von Hoff D, Mukkaram Ali M, et al. Preclinical development and clinical translation of a PSMA-targeted docetaxel nanoparticle with a differentiated pharmacological profile. Sci Transl Med. 2012;4(128):128 ra39.

5. Wong C, Stylianopoulos T, Cui J, et al. Multistage nanoparticle delivery system for deep penetration into tumor tissue. Proc Natl Acad Sci USA. 2011;108(6):2426-2431.

6. Zoabi N, Golani-Armon A, Zinger A, et al. The evolution of tumortargeted drug delivery: From the EPR effect to nano swimmers. Israel Journal of Chemistry. 2013;53(9-10):719-723.

7. Kirpotin DB, Drummond DC, Shao Y, et al. Antibody targeting of longcirculating lipidic nanoparticles does not increase tumor localization but does increase internalization in animal models. Cancer Res. 2006;66(13):6732-6740.

8. Han H, Davis ME. Single-antibody, targeted nanoparticle delivery of camptothecin. Mol Pharmacol. 2013;10(7):2558-2567.

9. Chahibi Y, Pierobon M, Akyildiz IF. Pharmacokinetic modeling and biodistribution estimation through molecular communication paradigm. IEEE Trans on Biomed Engg. 2015;62(10):2410-2420.

10. Chahibi Y, Akyildiz IF, Balasubramaniam S, et al. Molecular communication modeling of antibody drug delivery systems. IEEE Trans on Biomed Engg. 2015;62(7):1683-1695.

11. Sutton JT, Haworth KJ, Pyne-Geithman G, et al. Ultrasound-mediated drug delivery for cardiovascular disease. Expert Opinion on Drug Delivery. 2013;10(5):573-592. 
12. Coussios CC, Roy RA. Applications of acoustics and cavitation to noninvasive therapy and drug delivery. Annu Rev Fluid Mech. 2008;40:395-420.

13. Wang W1, Castro LA, Hoyos M, et al. Autonomous motion of metallic microrods propelled by ultrasound. ACS Nano. 2012;6(7):6122-6132.

14. Buttinoni I, Volpe G, Kümmel F, et al. Active Brownian motion tunable by light. J Phys Condens Matter. 2012;24(28):284129.

15. Ibele M1, Mallouk TE, Sen A. Schooling behavior of lightpowered autonomous micromotors in water. Angew Chem Int Ed. 2009;48(18):3308-3312.

16. Berg HC. Random Walks in Biology. USA: Pinceton Unviversity Press; 1993. p. $1-7$.

17. Berg HC, Purcell EM. Physics of chemoreception. Biophysics J. 1977;20(2):193-219.

18. Coley WB. II. Contribution to the knowledge of Sarcoma. Ann Surg. 1891;14(3):199-220.

19. Fialho AM, Bernardes N, Chakrabarty AM. Recent patents on live bacteria and their products as potential anticancer agents. Recent Pat Anti-Cancer Drug Discov. 2012;7(21):31-55.

20. Forbes NS. Engineering the perfect (bacterial) cancer therapy. Nat Rev Cancer. 2010;10(11):785-794.

21. Danino T, Lo J, Prindle A, et al. In vivo gene expression dynamics of tumor-targeted bacteria. ACS Synth Biol. 2012;1(10):465-470.

22. Baban CK, Cronin M, O’Hanlon D, et al. Bacteria as vectors for gene therapy of cancer. Bioeng Bugs. 2010;1(6):385-394.

23. Liu Y, Zhou M, Luo D, et al. Bacteria-mediated in vivo delivery of quantum dots into solid tumor. Biochem Biophys. Res. Commun. 2012;425(4):769-774.

24. Danino T, Prindle A, Kwong GA, et al. Programmable probiotics for detection of cancer in urine. Sci Transl Med. 2015;7(289):289ra84.

25. Heimann DM, Rosenberg SA. Continuous intravenous administration of live genetically modified salmonella typhimurium in patients with metastatic melanoma. J Immunother. 2003;26(2):179-180.

26. Toso JF, Gill VJ, Hwu P, et al. Phase I study of the intravenous administration of attenuated salmonella typhimurium to patients with metastatic melanoma. J Clin Oncol. 2002;20(1):142-152.
27. Nemunaitis J, Cunningham C, Senzer N, et al. Pilot trial of genetically modified, attenuated salmonella expressing the E. coli cytosine deaminase gene in refractory cancer patients. Cancer Gene Ther. 2003;10(10):737744.

28. Thornlow DN, Brackett EL, Gigas JM, et al. Persistent enhancement of bacterial motility increases tumor penetration. Biotechnol Bioeng. 2015;112(11):2397-2405.

29. Cohen I, Golding I, Kozlovsky Y, et al. Continuous and discrete models for cooperation in complex bacterial colonies. Fractals. 1999;7(3):235247.

30. Okaie Y, Nakano T, Hara T, et al. Cooperative target tracking by a mobile bionanosensor network. IEEE Trans Nanobioscience. 2014;3(3):267277.

31. Nakano T, Kobayashi S, Suda T, et al. Externally controllable molecular communication systems for pattern formation. USA: ACM; 2014.

32. Externally controllable molecular communication. IEEE J Sel Areas Commun. 2014;32(12):2417-2431.

33. Kasinskas RW, Forbes NS. Salmonella typhimurium specifically chemotax and proliferate in heterogeneous tumor tissue in vitro. Biotechnology and Bioengineering. 2006;94(4):710-721.

34. Charteris N, Khain E. Modeling chemotaxis of adhesive cells: Stochastic lattice approach and continuum description. New J Physics. 2014;16(2):1-12.

35. Lavrentovich MO, Nelson DR. Survival probabilities at spherical frontiers. Theor Popul Biol. 2015;102:26-39.

36. Deng B. Performance analysis of multiple simultaneous communications in bacterial nanonetworks. Finland: Master of Science Dissertation, Tampere University of Technology; 2015. p. 1-52.

37. Wei G, Bogdan P, Marculescu R. Bumpy rides: Modeling the dynamics of chemotactic interacting bacteria. IEEE J Sel Areas Commun. 2013;30(12):879-890.

38. Gillespie D. Exact stochastic simulation of coupled chemical reactions. $J$ Phy Chem. 1977;81(5):2340-2361.

39. Kleinrock L. Queuing Systems. USA: Wiley; 1975.

40. Anand S, Chandramouli R, Subbalakshmi KP. Cost of collaborative vs individual effort in social networks. SIAM; 2011. p. 1-11. 